\title{
Optic Neuropathy in Idiopathic Intracranial Hypertension
}

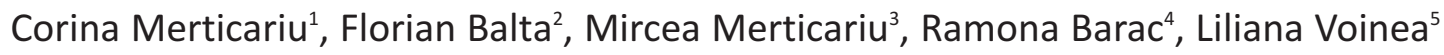

Victor Gomoiu Pediatric Hospital, Bucharest, Romania

${ }^{2}$ Vitreo-retinal Department, Emergency Eye Hospital and Clinic, Bucharest, Romania ${ }^{3}$ Carol Davila Nephrology Hospital, Bucharest, Romania

${ }^{4}$ Department of Ophthalmology, Emergency Eye Hospital and Clinic, Bucharest, Romania

University Emergency Hospital, Bucharest, Romania

${ }^{5}$ Department of Ophthalmology, University Emergency Hospital, Bucharest, Romania

\author{
Corresponding author: \\ Florian Balta, MD \\ Retina Clinic, No 85A \\ NicolaeCaranfil Street, sector 1 \\ Bucharest, Romania \\ E-mail: corina.taban@gmail.com
}

\section{ABSTRACT}

Adult patients who present with papilloedema and symptoms of raised intracranial pressure need urgent multidisciplinary assessment including neuroimaging, to exclude life-threatening causes. Where there is no apparent underlying cause for the raised intracranial pressure, patients are considered to have idiopathic intracranial hypertension (IIH).

Objectives: This review encompasses the newly revised diagnostic criteria for PTCS in adults and children and the role of obesity in the epidemiology, etiology, and management of $\mathrm{IH}$.Furthermore, this paper tries to offer a practical approach to assessing patients with papilloedema, the investigations and the subsequent management of patients with $\mathrm{IH}$.

Methodology: PubMed was used to search for $\mathrm{IIH}$ and Pseudotumor Cerebri. The vast majority of the literature consisted of cohort studies, case control studies, systematic reviews and other narrative reviews.

Conclusion: Idiopathic intracranial hypertension $(\mathrm{IIH})$, also known as primary Pseudotumor Cerebri syndrome (PTCS), is a condition of unknown etiology which affects primarily overweight, reproductive-aged women and causes increased intracranial pressure (ICP). This review discusses the recently revised diagnostic criteria for $\mathrm{IIH}$ for adults and children and the controversial issues in its diagnosis and management.

Key words: idiopathic intracranial hypertension, acetazolamide, pseudotumorcerebri

\section{INTRODUCTION}

Once thought to be rare, $\mathrm{IH}$ is becoming more common with an estimated incidence of 15-19 cases per 100000 among overweight or obese women ages 20-44. (1) This increasing incidence has been attributed to the growing obesity epidemic among adults. It most often affects obese or overweight adult females and can lead to blindness in up to $10 \%$ of patients, particularly if it is not recognized or treated promptly. (2) 


\section{Epidemiology}

Idiopathic intracranial hypertension $(\mathrm{IIH})$ is a disease that has a significant burden on the individual and on society. On average, the incidence of $\mathrm{IIH}$ in the general population is $0.5-1.0 / 100,000$ (3) and $3.5 / 100,000$ in young women aged 20 to 44 . (4)

The pathogenic mechanism underlining it is unknown and there is no sign of cerebral edema.

Obesity is a major risk factor, as the incidence of $\mathrm{IIH}$ is $13 / 100,000$ in women with $>10 \%$ over their optimal body mass index (BMI) and 19/100,000 in women with $>20 \% \%$ over their optimal body mass index (BMI). Moreso, $86 \%$ of patients with $\mathrm{IH}$ are obese and $12 \%$ overweight. Although it is mostly described as a disease affecting young overweight women, there is an increasing incidence in overweight men and children, parallelto the rising prevalence of obesity. $(1,5,6,7)$

In Romania, according to the latest statistics, $19.1 \%$ of the women aged 18 to 44 years are overweight, which is more than twice the value reported in 1980 when the rate of obesity in the general population was only $8.6 \%$. (8)

\section{Pathogenesis}

The pathogenesis of IIH is unknown. Although brain edema, increased cerebral blood volume, and increased CSF secretion have been postulated as IIH mechanisms (9) most attention has been focused on increased venous sinus pressure and decreased CSF absorption. Decreased CSF absorption by arachnoid villi has been demonstrated by radioisotope cisternography, although it is unclear whether it is secondary to compression of the arachnoid villi or by elevated ICP itself. (10)

\section{Diagnosis}

Idiopathic intracranial hypertension $(\mathrm{IIH})$ is a disorder that typically presents with headache and blurred vision and is diagnosed by the presence of papilledema and elevated intracranial pressure in the absence of infectious, vascular, or structural causes. Patients with IIH have normal levels of consciousness and functioning. (11)

Patients who present with papilloedema and symptoms of raised intracranial pressure need urgent multidisciplinary assessment including neuroimaging, to rule out life threatening conditions. Where there is no apparent underlying cause for the raised intracranial pressure, patients are considered to have idiopathic intracranial hypertension (IIH). (12)

At the moment $\mathrm{IIH}$ is confirmed, up to $90 \%$ of patients already have various degrees of visual impair- ment and 10 to $24 \%$ finally progress to permanent severe visual impairment. Visual loss can occur any time along the course of the disease and is usually insidious. Central vision is not affected until late so the visual loss is usually unnoticed by the patient, up to when it is very advanced.

Furthermore, the diagnosis is usually delayed as medical knowledge about IIH is scarce and many doctors from different clinical subspecialties are consulted before the patient is finally diagnosed and treated. Visual prognosis depends on the speed of diagnosis and on a thorough follow-up.

As the vast majority of patients with IIH complain of impairing headaches, therapeutic attitude towards IIH varies. Although it is the only irreversible damage of the disease, visual loss usually occurs gradually and silently, only a small percent of patients having a high risk of rapid visual loss.

Visual acuity loss in IIH can be caused by both outer retinal changes and optic neuropathy. Retinal changes include chorioretinal folds, hyperopic shift, hemorrhages, macular edema, subretinal fluid,or, rarely, subretinalneovascularization. (13)

Distinguishing outer retinal changes from optic neuropathy as the cause of decreased visual acuity is important, because outer retinal changes in the macula affecting visual acuity may be largely reversible, whereas fulminant $\mathrm{IH}$ with optic neuropathy and neuronal loss may require more aggressive management, such as immediate surgical intervention, to prevent further vision loss and irreversible blindness. $(14,15,16,17,18)$

Diagnostic criteria for adult IIH- Adapted from the 2013 revised diagnostic criteria for IIH: (19)

- Papilloedema;

- Normal neurological examination except for cranial nerve abnormalities;

- Neuroimaging: Normal brain parenchyma without hydrocephalus, mass or structural lesion and no abnormal meningeal enhancement or venous sinus thrombosis on MRI and MR venography; if MRI is unavailable or contraindicated, contrastenhanced CT may be used;

- Normal CSF composition;

- Elevated CSF opening pressure $(\geq 25 \mathrm{~cm} \mathrm{H} 2 \mathrm{O})$ in a properly performed lumbar puncture.

A diagnosis of IIH is definite in patients fulfilling A-E; the diagnosis is probable if $A-D$ are met but the CSF pressure is lower than specified.

The diagnosis can be difficult and the consequences of error can lead either to the neglect of a serious treatable cause of raised intracranial pressure, blind- 
ness or inappropriate treatment of patients who do not have $\mathrm{IIH}$. Although there is insufficient literature to generate an evidence-based management strategy for $\mathrm{IIH}$,experienced clinicians can manage it well. (20) (fig. 1)

\section{Papilloedema}

Patients generally present to the emergency department after an optometrist or family doctor detects papilloedema. They may or may not have other symptoms. Because papilloedema indicates potentially serious underlying disease, the purpose of the visit is to recognise and confirm the presence of papilloedema and arrange appropriate onward investigations. (12)

When there is papilloedema with symptoms and signs of raised intracranial pressure, it is important to obtain a thorough past history and system screen to identify treatable causes:

Secondary causes of raised intracranial pressure for exclusion to diagnose $\mathrm{IH}$ :

- Secondary causes of raised intracranial pressure;

- Venous sinus thrombosis;

- Anaemia;

- Obstructive sleep apnoea;

- Drug-related;

- CSF hyperproteinaemia/hypercellularity, for example, spinal cord tumour / meningitis / GuillainBarré syndrome/subarachnoid haemorrhage;
- Renal failure;

- Endocrine diseases, for example, Addison's/ Cushing's/hypothyroidism;

- Drugs associated with pseudotumour cerebri: (12)

- Tetracycline/minocycline/doxycycline;

- Corticosteroids;

- Beclometasone;

- Nitrofurantoin;

- Tamoxifen;

- Sulphonamides, for example, trimethoprim;

- Ciclosporin;

- Nalidixic acid;

- Non-steroidal anti-inflammatory drugs;

- Vitamin A excess and retinoids;

- Cimetidine;

- Lithium;

- Depo Provera.

\section{Neuroimaging}

Having identified papilloedema, it is essential to record blood pressure to exclude malignant hypertension. Patients then need urgent neuroimaging: this has two purposes, to identify any space-occupying lesion and to exclude a venous sinus thrombosis. The preferred imaging method is an MR scan of the head and orbits with intravenous contrast and MR venogram; these should, ideally, include fat suppression sequences, as these better define the intraorbital optic

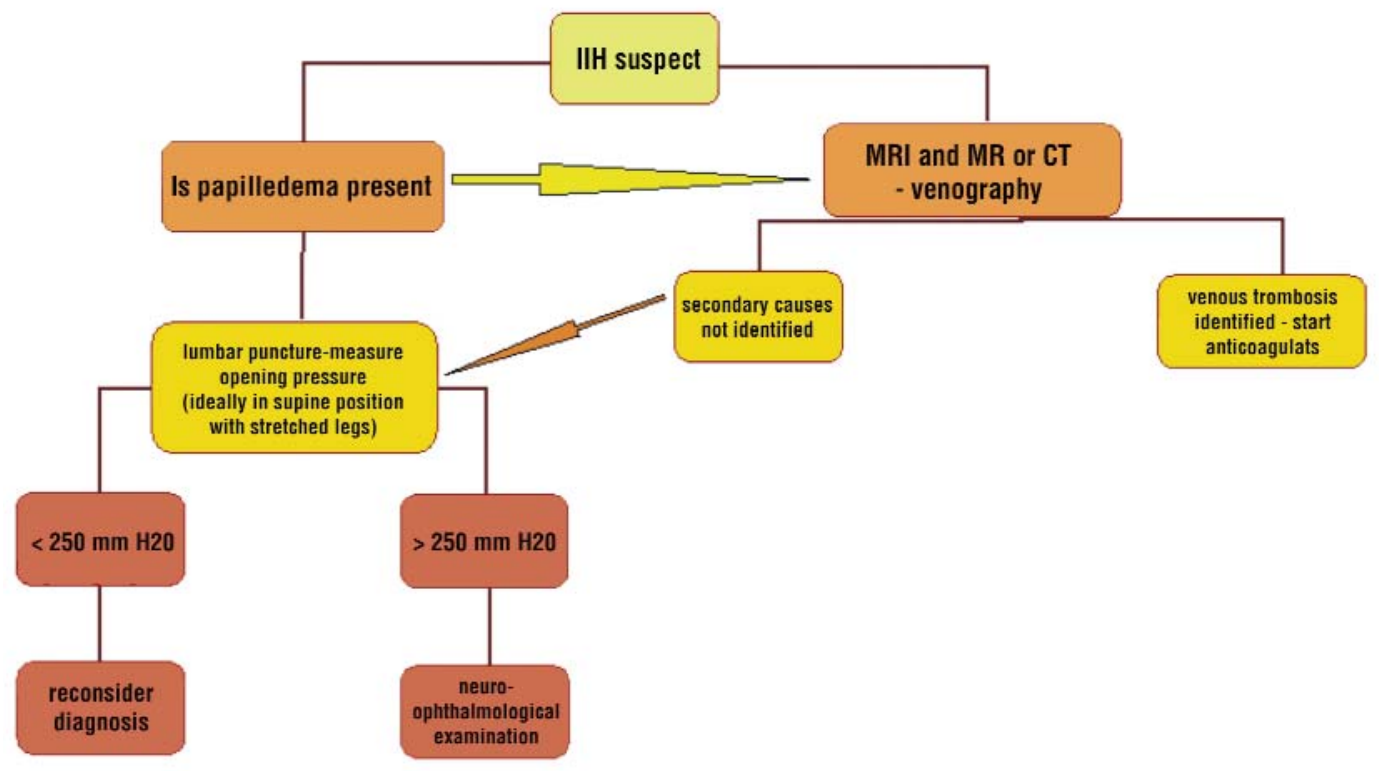

Figure 1 - Proposal of a diagnostic work-up and strategy in patients with possible idiopathic intracranial hypertension [adapted from Jensen $\mathrm{RH}$ et al (31)] 
nerves. However, if this is not readily available, a CT head scan with a CT venogram will exclude most space-occupying lesions, cerebral venous sinus thrombosis and Chiari malformation. There are several possible radiological signs of $\mathrm{IIH}$, although none is pathognomonic. There may be an empty sella, a partially empty sella, decreased pituitary height or transverse sinus narrowing. In the orbits, the optic nerve sheath complex may be enlarged, the posterior globe flattened and occasionally the optic nerve head protruded. (21)

\section{Lumbar puncture}

After excluding a structural intracranial lesion, patients require a lumbar puncture, performed with the patient in the lateral decubitus position. Whiteley'sclinical study found the normal range for lumbar CSF opening pressure was 10-25 cm CSF (95\% reference interval); but some normal subjects had opening pressures of up to $28 \mathrm{~cm}$ CSF. (22) In adults and children the assessment of average CSF pressure over more than 20 minutes, 'steady state', is reported to be more reliable than a single opening pressure measurement using the height of a fluid column. $(23,24,25)$

Diagnostic errors resulted in overdiagnosis of $\mathrm{IIH}$ in $39.5 \%$ of patients referred for presumed $\mathrm{IH}$ to a neuro-ophthalmology service of a tertiary center and prompted unnecessary tests, invasive procedures, and missed diagnoses. The most common errors were inaccurate ophthalmoscopic examination in headache patients and thinking biases, reinforcing the need for rapid access to specialists with experience in diagnosing optic nerve disorders. Indeed, the high prevalence of primary benign headaches and obesity in young women often leads to costly and invasive evaluations for presumed IIH. (26)

As emphasized in the DEER (diagnostic error evaluation research) classification, diagnostic errors can result from various mistakes along the course of a patient evaluation. Most errors resulted from inability to perform an accurate physical examination (i.e., ocular fundus examination) and from the difficulty in deviating from a previously suspected diagnosis (based on the intuitive presumption that obese women with headaches must have $\mathrm{IIH}$ ). In a few cases, isolated radiologic findings raised a concern for $\mathrm{IIH}$, or moderately elevated CSF opening pressure in the absence of papilledema or sixth nerve palsies prompted the wrong diagnosis. (26)

There are a number of relatively simple ways to reduce the risk of misdiagnosis.

Studies suggest that characteristic MRI findings include empty sellaturcica, decreased pituitary gland size, optic nerve tortuosity, perioptic subarachnoid space enlargement, posterior globe flattering, and intraocular protrusion of the optic nerve head. In these patients, a detailed fundoscopic examination by an experienced ophthalmologist or neuro-ophthalmologist, should be enough to either confirm papilloedema and lead to further investigations, or refute it and render other investigations useless. (27)

Apart form this, recent findings suggest that on optical coherence tomography (OCT), increased retinal nerve fiber layer and macular thickness may be observed in children with IIH compared with controls. The retinal nerve fiber layer thickness seems to coincide with the severity of papilledema and may be more sensitive than fundoscopy for detecting optic nerve head elevation. (fig. 2) (28) Research on ultrasound of the optic nerve shows increased size of the
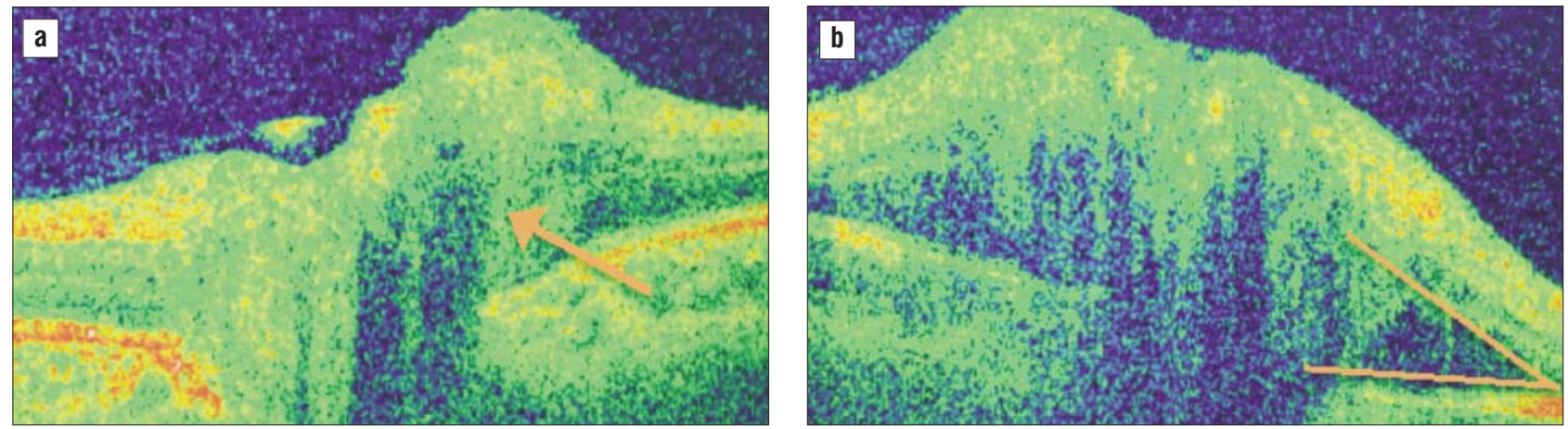

Figure 2 - Optical coherence tomography (cross-sectional image) through an optic disc with drusen and papilloedema

(a) Optical coherence tomogram (cross-sectional image) through an optic disc with drusen showing the typically irregular tissue underlying

the elevated surface of the disc with no extension of the sub-retinal hyporeflective layer (arrows) beyond the optic nerve head; (b) 0ptical coherence tomogram (cross-sectional image) of a papilloedematous optic disc showing a smoothly elevated disc with underlying hyporeflective fluid extending beyond the disc into the sub-retinal space in a 'lazy V pattern' (illustrated by lines) [adapted from Choi SS et al (28)] 
optic nerve sheath diameter in pediatric IIH patients, and this may correlate with increased opening pressure on lumbar puncture. (29)

\section{Other investigations}

Patients in whom the intracranial hypertension is not truly idiopathic but has an identifiable etiology (otitis media, dural sinus thrombosis, systemic lupus erythematosus, neck injury, metastatic disease, nephrotic syndrome or arteriovenous malformations) should be excluded.

\section{Evolution of optic neuropathy in IIH}

Disease evolution and outcomes in IIH are poorly characterised and are currently being evaluated in the National IIH: LIFE study. The following patient types were noted: (12)

- Those who rapidly lose vision at diagnosis over days to weeks (rare but vital to identify early);

- Those whose disease resolves following diagnosis, over weeks to months, occasionally after a single lumbar puncture (rare);

- Those at lower risk of visual loss who develop chronic disease with small fluctuations in disease activity, frequently with weight changes (the majority);

- Those in disease remission and off treatment.

Diagnosing and monitoring optic neuropathy in IIH is the clinical priority for all patients. Visual function can be fully assessed onlyby measuring visual acuity, colour vision and visual fieldtesting. Pupillary reflex tests and slit-lamp examination should be performend by an experienced ophthalmologistor a neuroophthalmologist. According to recent studies and improvement in available software, opticalcoherence tomography (OCT) is proving to be useful in aiding the diagnosis and followup of papilledema and optic neuropathy. (table 1)

\section{Treatment and management}

Different treatment options are available. Conservative measures focus on weight loss, either through diet or through bariatric surgery. Pharmacological therapy includes diuretics. Surgery is reserved for cases reluctant to medical treatment and involve optical nerve fenestration, CSF divergence and venous sinus stenting. It should only be advised in cases with malignant intracranial hypertension or rapid progression, if there is severe sight threatening. Surgical treatment did not prove effective in alleviating headaches. (30) According to symptom severity, the majority of doctors use a stratified treatment strategy for idiopathic intracranial hypertension. (fig. 3) Proposal of a stratified treatment strategy for idiopathic intracranial hypertension according to symptom severity. (31)

\section{Management of acute visual loss in IIH}

In high-risk patients with impending visual loss, some form of CSF divergence can be sight-saving and can sometimes lead to visual recovery. Options include a ventriculoperitoneal, lumboperitoneal shunt, where a catheter is inserted into the subarachnoid space either in the lateral ventricules or at the lumbar spine between two vertebrae and fed around the oblique muscles under the skin into the peritoneum. Less commonly, a ventriculoatrialor a ventriculopleural shunt can divert CSF from the lateral ventricle to the atrium or the pleura. (32) While the shunt is working, weight reduction is advised, to put their disease into remission. Additionally, shunting exclusively to treat headache, is almost never recommend as this continues in most patients postoperatively (68\% at 6 months and $79 \%$ at 2 years). Also, postoperative lowpressure headache occurs in $28 \%$. Although shunting seems effective in alleviating acute symptoms of $\mathrm{IH}$, significant complications include blockage of drainage,

Table 1 - Diagnosing and monitoring optic neuropathy in IIH [adapted from Susan P Mollan et al. (13)]

\begin{tabular}{|c|c|}
\hline Visual acuity & $\begin{array}{l}\text { Test each eye separately (using a Snellen's or logMar chart) for the best corrected distance visual acuity } \\
\text { (with glasses or use a pinhole to record improvement after correcting for refractive error) }\end{array}$ \\
\hline Colour vision & Test each eye individually with pseudoisochromatic plates (such as Ishihara's plates) \\
\hline Pupil examination & Exclude a relative afferent pupillary defect and oculosympathetic palsy (Horner's syndrome) \\
\hline Dilated fundus examination & $\begin{array}{l}\text { Document optic nerve head AND macular findings using a slit lamp, to exclude intraocular inflammation causing } \\
\text { bilateral disc edema }\end{array}$ \\
\hline OCT & $\begin{array}{l}\text { Quantify the degree of neuropathy by measuring the width of the CGL-IPL complex } \\
\text { (according to the lowa Reference Algorithm) }\end{array}$ \\
\hline
\end{tabular}




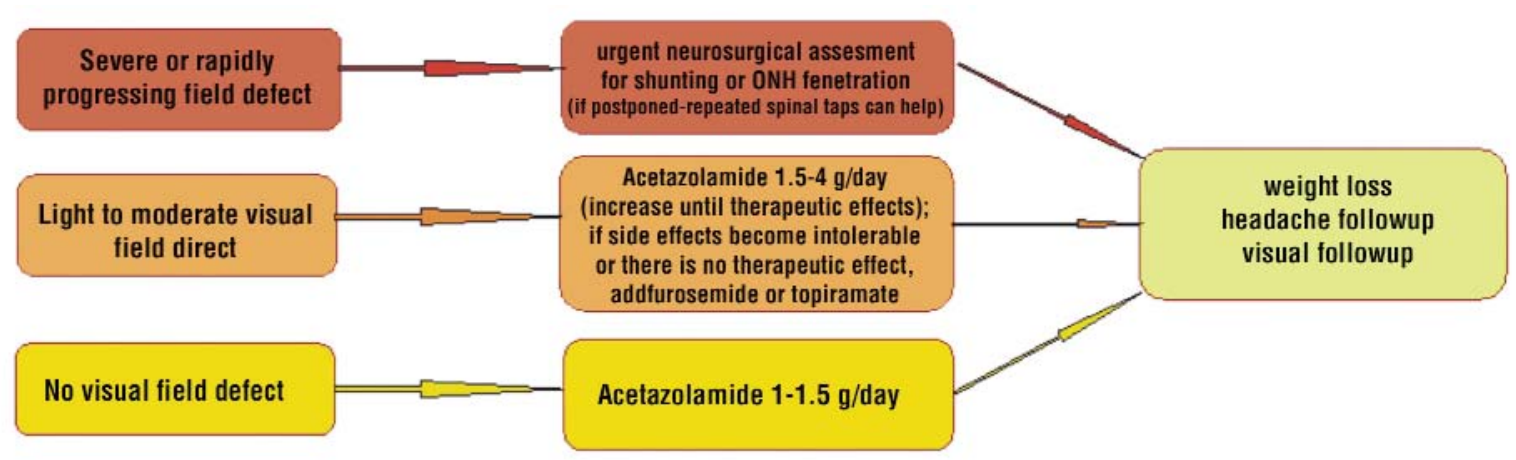

Figure 3 - Proposal of a stratified treatment strategy for idiopathic intracranial hypertension according to symptom severity [adapted from Jensen $\mathrm{RH}$ et al (31)]

infections, intracranial low-pressure, chronic abdominal and back pain. Moreso, over half the patients need shunt revision, and one-third need multiple revisions. (32) Optic nerve sheath fenestration is an alternative for protecting the vision; however, it has little effect on the overall intracranial pressure and can lead to visual loss in inexperienced hands.In situations of acute, severe visual loss, the combination of oral or IV acetazolamide and IV methylprednisolone $15 \mathrm{mg} / \mathrm{kg}$ can be used when surgery is not immediately available. (33)

\section{Pharmacological management}

Carbonic anhydrase inhibitors, such as acetazolamide, can provide symptomatic relief of raised intracranial pressure, although there is no evidence to support their long term benefit. The maximum dose is up to $2 \mathrm{~g}$ /daily; usually, up to $1 \mathrm{~g}$ daily is adequate, depending on body weight. Common dose-related side effects include Gl upset, paresthesias involving the lips, fingers, and toes, anorexia, and electrolyte imbalance (metabolic acidosis).When the side effects become intolerable, the dose is lowered or acetazolamide is replaced or combined with furosemide. Topiramate, an antiepileptic medication which has secondary carbonic anhydrase activity, may be used as a second-line agent. Topiramate use in IIH is relatively new and has the added benefit of appetite suppression and weight loss in many patients. It is an excellent medication for chronic daily headache and it has been used safely for years in patients with epilepsy. Although the optimal duration of treatment is unknown, some experts recommend that treatment is continued for at least 6 months after visual status, and optic nerve appearance stabilize before tapering off medications. (34)

Studies so far, could neither support nor deny the longtermbenefit of acetazolamide or any other type of medical therapy in the treatment of $\mathrm{IIH} .(35,36)$

\section{Conservative management}

Weight loss is currently the only proven diseasemodifying treatment for all overweight patients. Recent studies highlighted that a $15 \%$ reduction of body weight, using a low calorie meal replacement liquid diet for 3 months, significantly reduced intracranial pressure, papilloedema and headaches. $(37,38)$ Furthermore, current studies suggest that bariatric surgery might have an important role in the long-term treatment of IIH. $(39,40)$

\section{CONCLUSION}

Idiopathic intracranial hypertension is associated with severe morbidity due to the associated optic neuropathy and severe headaches. This review summarizes the relevant articles on the diagnostic tools used in evaluation management of $\mathrm{IH}$, highlighting the importance of early recognition and management of optic neuropathy to prevent permanent visual loss.

Steady state CSF pressure assessment, rather than measuring CSF opening pressure as well as expert opinion on high-quality MRI and MRV is often helpful. Before subjecting a patient to invasive investigations, on the basis of possible papilloedema, it is recommended that he be examined by an expert ophthalmologist, also using ultrasonic and OCT imaging to support the diagnosis.

By organizing a functional pathway and easy access to medical visits and follow-up, diagnosis can be effectively carried out by a team of professionals comprising an ophthalmologist, a neurologist, a radiologist and a nutritionist and treatment can be adjusted according to individual needs. Therefore, raising awareness and further research into how to diagnose and treat IIH is of the utmost importance. 


\section{REFERENCES}

1. Durcan FJ, Corbett JJ, Wall M. The incidence of pseudotumor cerebri. Population studies in lowa and Louisiana. 1988. Arch Neurol. 1988 Aug;45(8):875-7.

2. Ball AK, Clarke CE. Idiopathic intracranial hypertension. Lancet Neurol. 2006 May;5(5):433-42.

3. Carta A, Bertuzzi F, Cologno D, Giorgi C, Montanari E, Tedesco S Idiopathic intracranial hypertension (pseudotumor cerebri): descriptive epidemiology, clinical features, and visual outcome in Parma, Italy, 1990 to 1999. Eur J Ophthalmol. 2004 Jan-Feb;14(1):48-54.

4. Andrews LE, Liu GT, Ko MW. Idiopathic intracranial hypertension and obesity. Horm Res Paediatr. 2014;81(4):217-25.

5. Radhakrishnan K, Ahlskog JE, Cross SA, Kurland LT, O'Fallon WM. Idiopathic intracranial hypertension (pseudotumor cerebri). Descriptive epidemiology in Rochester, Minn, 1976 to 1990. Arch Neurol. 1993 Jan:50(1):78-80.

6. Bruce BB, Preechawat P, Newman NJ, Lynn MJ, Biousse V. Racial differences in idiopathic intracranial hypertension. Neurology. 2008 Mar 11;70(11):861-7. doi: 10.1212/01.wnl.0000304746.92913.dc.

7. Bruce BB, Kedar S, Van Stavern GP, Monaghan D, Acierno MD, Braswell RA, et al. Idiopathic intracranial hypertension in men. Neurology. 2009 Jan 27;72(4):304-9. doi: 10.1212/01.wnl. 0000333254.84120.f5. Epub 2008 0ct 15

8. Overweight and Obesity. http://ec.europa.eu/eurostat/statisticsexplained/index.php/Overweight_and_obesity_-_BMI_statistics. [Online] nov 29, 2016.

9. Johnston IH, Duff J, Jacobson EE, Fagan E. Asymptomatic intracranial hypertension in disorders of CSF circulation in childhood--treated and untreated. Pediatr Neurosurg. 2001 Feb;34(2):63-72.

10. Liu GT, Volpe NJ, Galetta S. Neuro-ophthalmology: diagnosis and management. Philadelphia: Saunders; 2001.

11. Brara SM, Koebnick C, Porter AH, Langer-Gould A. Pediatric idiopathic intracranial hypertension and extreme childhood obesity. J Pediatr. 2012 Oct;161(4):602-7. doi: 10.1016/j.jpeds.2012.03.047. Epub 2012 May 26.

12. Mollan SP, Markey KA, Benzimra JD, Jacks A, Matthews TD, Burdon $M A$, et al. A practical approach to, diagnosis, assessment and management of idiopathic intracranial hypertension. Pract Neurol. 2014 Dec;14(6):380-90. doi: 10.1136/practneurol-2014-000821. Epub 2014 May 8.

13. Chen JJ, Thurtell MJ, Longmuir RA, Garvin MK, Wang JK, Wall M, et al. Causes and Prognosis of Visual Acuity Loss at the Time of Initial Presentation in Idiopathic Intracranial Hypertension. Invest Ophthalmol Vis Sci. 2015 Jun;56(6):3850-9. doi: 10.1167/iovs.1516450.

14. Hoye VJ 3rd, Berrocal AM, Hedges TR 3rd, Amaro-Quireza ML. Optical coherence tomography demonstrates subretinal macular edema from papilledema. Arch Ophthalmol. 2001 Sep;119(9):1287-90.

15. Akova YA, Kansu T, Yazar Z, Atabay C, Karagöz Y, Duman S. Macular subretinal neovascular membrane associated with pseudotumor cerebri. J Neuroophthalmol. 1994 Dec;14(4):193-5.

16. Jamison RR. Subretinal neovascularization and papilledema associated with pseudotumor cerebri. Am J Ophthalmol. 1978 Jan;85(1):78-81.

17. Rush JA. Pseudotumor cerebri: clinical profile and visual outcome in 63 patients. Mayo Clin Proc. 1980 Sep;55(9):541-6.

18. Schirmer CM1, Hedges TR 3rd. Mechanisms of visual loss in papilledema. Neurosurg Focus. 2007;23(5):E5

19. Friedman DI, Liu GT, Digre KB. Revised diagnostic criteria for the pseudotumor cerebri syndrome in adults and children. Neurology. 2013 Sep 24;81(13):1159-65. doi: 10.1212/WNL.0b013e3182a55f17. Epub 2013 Aug 21.

20. Wright BL, Lai JT, Sinclair AJ. Cerebrospinal fluid and lumbar puncture: a practical review. J Neurol. 2012 Aug;259(8):1530-45. doi: 10.1007/s00415-012-6413-x. Epub 2012 Jan 26.

21. Wall M. Idiopathic intracranial hypertension. Neurol Clin. 2010 Aug;28(3):593-617. doi: 10.1016/j.ncl.2010.03.003
22. Whiteley W, Al-Shahi R, Warlow CP, Zeidler M, Lueck CJ. CSF opening pressure: reference interval and the effect of body mass index. Neurology. 2006 Nov 14;67(9):1690-1.

23. Krishnakumar D, Pickard JD, Czosnyka Z, Allen L, Parker A. Idiopathic intracranial hypertension in childhood: pitfalls in diagnosis. Dev Med Child Neurol. 2014 Aug;56(8):749-55. doi: 10.1111/dmcn.12475. Epub 2014 May 22.

24. Schuhmann MU, Sood S, McAllister JP, Jaeger M, Ham SD, Czosnyka $Z$, et al. Value of overnight monitoring of intracranial pressure in hydrocephalic children. Pediatr Neurosurg. 2008;44(4):269-79. doi: 10.1159/000131675. Epub 2008 May 15

25. Marmarou A, Bergsneider M, Klinge P, Relkin N, Black PM. The value of supplemental prognostic tests for the preoperative assessment of idiopathic normal-pressure hydrocephalus. Neurosurgery. 2005 Sep;57(3 Suppl):S17-28; discussion ii-v.

26. Fisayo A, Bruce BB, Newman NJ, Biousse V. Overdiagnosis of idiopathic intracranial hypertension. Neurology. 2016 Jan 26;86(4):34150. doi: 10.1212/WNL.0000000000002318. Epub 2015 Dec 30.

27. Ducros A, Biousse V. Headache arising from idiopathic changes in CSF pressure. Lancet Neurol. 2015 Jun;14(6):655-68. doi: 10.1016/ S1474-4422(15)00015-0.

28. Choi SS, Zawadzki RJ, Keltner JL, Werner JS. Changes in cellular structures revealed by ultra-high resolution retinal imaging in optic neuropathies. Invest Ophthalmol Vis Sci. 2008 May;49(5):2103-19. doi: 10.1167/iovs.07-0980.

29. Gilbert AL, Heidary G. Update on the evaluation of pediatric idiopathic intracranial hypertension. Curr Opin Ophthalmol. 2016 Nov;27(6):493497.

30. Yri HM1, Wegener M, Sander B, Jensen R. Idiopathic intracranial hypertension is not benign: a long-term outcome study. J Neurol. 2012 May;259(5):886-94. doi: 10.1007/s00415-011-6273-9. Epub 2011 Oct 19.

31. Jensen $R H$, Radojicic A, Yri $H$. The diagnosis and management of idiopathic intracranial hypertension and the associated headache. Ther Adv Neurol Disord. 2016 Jul;9(4):317-26. doi: 10.1177/ 1756285616635987. Epub 2016 Mar 21.

32. Sinclair AJ, Kuruvath S, Sen D, Nightingale PG, Burdon MA, Flint G. Is cerebrospinal fluid shunting in idiopathic intracranial hypertension worthwhile? A 10-year review. Cephalalgia. 2011 Dec;31(16):162733. doi: 10.1177/0333102411423305. Epub 2011 Oct 3.

33. Liu GT, Glaser JS, Schatz NJ. High-dose methylprednisolone and acetazolamide for visual loss in pseudotumor cerebri. Am J Ophthalmol. 1994 Jul 15;118(1):88-96.

34. Johnston IH, Duff J, Jacobson EE, Fagan E. Asymptomatic intracranial hypertension in disorders of CSF circulation in childhood--treated and untreated. Pediatr Neurosurg. 2001 Feb;34(2):63-72.

35. Ball AK, Howman A, Wheatley K, Burdon MA, Matthews T, Jacks AS, et al. A randomised controlled trial of treatment for idiopathic intracranial hypertension. J Neurol. 2011 May;258(5):874-81. doi: 10.1007/ s00415-010-5861-4. Epub 2010 Dec 16.

36. Friedman DI. Cerebral venous pressure, intra-abdominal pressure, and dural venous sinus stenting in idiopathic intracranial hypertension. J Neuroophthalmol. 2006 Mar;26(1):61-4

37. Sinclair AJ, Burdon MA, Nightingale PG, Ball AK, Good P, Matthews $T D$, et al. Low energy diet and intracranial pressure in women with idiopathic intracranial hypertension: prospective cohort study. BMJ. 2010 Jul 7;341:c2701. doi: 10.1136/bmj.c2701.

38. Newborg B. Pseudotumor cerebri treated by rice reduction diet. Arch Intern Med. 1974 May;133(5):802-7.

39. Fridley J, Foroozan R, Sherman V, Brandt ML, Yoshor D. Bariatric surgery for the treatment of idiopathic intracranial hypertension. J Neurosurg. 2011 Jan;114(1):34-9. doi: 10.3171/2009.12.JNS09953. Epub 2010 Jan 22.

40. Jolly K, Lewis A, Beach J, Denley J, Adab P, Deeks JJ, et al. Comparison of range of commercial or primary care led weight reduction programmes with minimal intervention control for weight loss in obesity: lighten Up randomised controlled trial. BMJ. 2011 Nov 3;343:d6500. doi: 10.1136/bmj.d6500. 